\title{
THE ENVELOPE OF HOLOMORPHY OF A TRUNCATED TUBE
}

\author{
ZAIFEI YE
}

(Communicated by Clifford J. Earle, Jr.)

Abstract. A counterexample is constructed to show an essential difference between local and global CR extensions in tube domains.

In $I^{4}=:\left\{(z, w) \in \mathbb{C}^{2}|| \operatorname{Re} z|<1,| \operatorname{Im} z|<1,| \operatorname{Re} w|<1,| \operatorname{Im} w \mid<1\right\}$, let us consider the following type of truncated tube domain $\Omega_{h} \subset \mathbb{C}^{2}$ :

$$
\Omega_{h}=:\left\{(z, w) \in I^{4} \mid \operatorname{Re} w>h(\operatorname{Re} z)\right\},
$$

with boundary

$$
\Sigma_{h}=b \Omega_{h}=:\left\{(z, w) \in I^{4} \mid \operatorname{Re} w=h(\operatorname{Re} z)\right\},
$$

where $h$ satisfies $h \in C^{\infty}(I), I=:(-1,1), h(0)=0, h^{\prime}(0)=0$, and $h$ attains both positive and negative values in any neighborhood of 0 . It is clear from Trepreau's theorem [T] that any CR function on $\Sigma_{h}$ extends holomorphically to at least one side, but Trepreau's theorem does not indicate to which side. In the situation described above, due to the changes of sign of $h$, one might have expected that every CR function extends to a full neighborhood of 0 as would happen in the case of an unlimited tube. Baouendi and Rothschild suggested that one look for an example where the extendability to both sides fails. We present such an example in this note.

Proposition. There exists a function $h$ as described above such that the envelope of holomorphy of $\Omega_{h}$ does not contain a neighborhood of 0 .

In fact, we obtain a pseudoconvex domain (putting $z=x+i y$ )

$$
\widetilde{\Omega}=:\left\{(z, w) \in I^{4} \mid \operatorname{Re} w>\phi(x, y)\right\}
$$

with $\phi \in C^{\infty}, \phi(0)=0$, which contains some $\Omega_{h}$ as above. Hence, there are holomorphic functions in $\Omega_{h}$ which do not extend holomorphically to a neighborhood of 0 . It implies the failure of the extendability of CR functions on $\Sigma_{h}$ to both sides. It is routine to check that $\widetilde{\Omega}$ is pseudoconvex if and only

Received by the editors October 12, 1989 and, in revised form, November 29, 1990.

1980 Mathematics Subject Classification (1985 Revision). Primary 32D15, 32A07; Secondary $31 \mathrm{~A} 05$.

Key words and phrases. CR function, pseudoconvexity, subharmonic function. 
if $\phi$ is subharmonic on $I^{2}=:\{z=x+i y|| x|<1| y \mid,<1\}$. The proposition, therefore, follows from:

Lemma. There is a $C^{\infty}$ subharmonic function $\phi$ defined in $\overline{I^{2}}$ satisfying

(i) $\phi(0)=0$.

(ii) There are two sequences of positive numbers $\left\{a_{n}\right\},\left\{b_{n}\right\}$ so that $a_{n} \rightarrow 0$, $b_{n} \rightarrow 0$ as $n \rightarrow \infty$, and for all $|y|<1, n \in \mathbb{N}$,

$$
\phi\left( \pm a_{n}+i y\right)<0, \quad \phi\left( \pm b_{n}+i y\right)>0 .
$$

(iii) There exists an $h \in C^{\infty}(I)$ so that $h(0)=h^{\prime}(0)=0$,

$$
h(x) \geq \max _{y \in I} \phi(x+i y), \quad \forall x \in I \text { and } h\left( \pm a_{n}\right)<0 .
$$

Proof. The basic building blocks for constructing $\phi$ are the following subharmonic functions $\psi_{n} \in C^{\infty}\left(I \times\left[-2^{n+1}, 2^{n+1}\right]\right)$ satisfying

(1) $-1 \leq \psi_{n}(x, y) \leq-\frac{1}{2}$, for $|x|<\frac{1}{8}$,

(2) $\frac{1}{2} \leq \psi_{n}(x, y) \leq 1$, for $|x|=\frac{1}{4}$,

(3) $\psi_{n}(x, y)=0$, for $|x|>\frac{3}{8}$.

The existence of such $\psi_{n}$ is a simple consequence of the Runge approximation theorem. As we know, there is an entire function $g$ such that

$$
\begin{array}{ll}
\left|g(z)+\frac{3}{4}\right|<\frac{1}{8} & \text { for }|\operatorname{Re} z|<\frac{1}{8},|\operatorname{Im} z| \leq 2^{n+2}, \\
\left|g(z)-\frac{3}{4}\right|<\frac{1}{8} & \text { for }|\operatorname{Re} z|=\frac{1}{4},|\operatorname{Im} z| \leq 2^{n+2}, \\
\left|g(z)+\frac{3}{4}\right|<\frac{1}{8} & \text { for } \frac{3}{8} \leq|\operatorname{Re} z| \leq \frac{3}{2},|\operatorname{Im} z| \leq 2^{n+2} .
\end{array}
$$

Then, we put

$$
\psi_{n, 0}(x, y)=: \begin{cases}\operatorname{Re} g(z), & \text { if }|x|<\frac{1}{4},|y|<2^{n+2}, \\ \max \{0, \operatorname{Re} g(z)\}, & \text { if } \frac{1}{4} \leq|x|<\frac{3}{2},|y|<2^{n+2} .\end{cases}
$$

Finally, $\psi_{n}$ is obtained by smoothing $\psi_{n, 0}$ (by convolution). Now, we set

$$
\phi(x, y)=: \begin{cases}\varepsilon_{n} \psi_{n}\left(2^{n+1}\left(|x|-\frac{3}{2^{n+1}}\right), 2^{n+1} y\right), & \frac{1}{2^{n}} \leq|x|<\frac{1}{2^{n-1}}, n=1,2, \ldots, \\ 0, & \text { otherwise, }\end{cases}
$$

where $\varepsilon_{n}>0$. At the same time, we define

$$
h(x)=: \begin{cases}\varepsilon_{n} h_{n}\left(2^{1+n}\left(|x|-\frac{3}{2^{n+1}}\right)\right), & \frac{1}{2^{n}} \leq|x|<\frac{1}{2^{n-1}}, n=1,2, \ldots, \\ 0, & \text { otherwise, }\end{cases}
$$

where $h_{n} \in C_{0}^{\infty}(I), \max _{y \in\left[-2^{n+1}, 2^{n+1}\right]} \psi_{n}(x, y) \leq h_{n}(x)<2\left\|\psi_{n}\right\|_{L^{\infty}\left(I \times\left[-2^{n+1}, 2^{n+1}\right]\right)}$, $\forall x \in I, h_{n}(0)<0$. Then, one can see that $\phi$ satisfies all the required properties in the lemma provided that $\varepsilon_{n}$ is chosen to be very small, say

$$
\begin{aligned}
\varepsilon_{n}=e^{-2^{n}} / \max \left\{1,\left\|\psi_{n}\right\|_{\mathbb{C}^{n}},\left\|h_{n}\right\|_{\mathbb{C}^{n}}\right\}, \\
\text { where }\|f\|_{\mathbb{C}^{n}}=\max _{\substack{p+q \leq n, p, q \geq 0 \\
|x| \leq 1,|y| \leq 2^{n+1}}}\left|\frac{\partial^{p+q} f}{\partial x^{p} \partial y^{q}}(x, y)\right|,
\end{aligned}
$$


for any smooth function $f$ defined on $I \times\left[-2^{n+1}, 2^{n+1}\right]$.

Remark. The oscillation of $h$ is an important factor in this example. One can check that if $h(x)>0$ for $x>0$ and $h(x)<0$ for $x<0$ then every CR function on $\sum_{h}$ in a neighborhood of 0 extends holomorphically to both sides, regardless how flat $h$ may be (see Theorem 1.1 in [BT]). Notice also that if $h$ is odd and nonlinear, it follows from Trepreau's theorem and by symmetry that every CR function on $\sum_{h}$ can be holomorphically extended to both sides.

\section{ACKNOWLEDGMENT}

I wish to thank Professors Baouendi and Rothschild for pointing out a mistake in the previous version of the paper.

\section{REFERENCES}

[BT] M. S. Baouendi and F. Treves, A microlocal version of Bochner's tube theorem, Indiana Univ. Math. J. 31 (1982), 885-895.

[T] J. M. Trepreau, Sur le prolongement holomorphe des fonctions $C R$ definis sur une hypersurface Réelle de classe $C^{2}$ dans $\mathbb{C}^{n}$, Invent. Math. 83 (1986), 583-592.

Department of Mathematics, University of Wisconsin at Madison, Madison, WisconSIN 53706

Current address: Department of Mathematics, University of California at San Diego, La Jolla, California 92093 